Stud. Univ. Babeş-Bolyai Math. 66(2021), No. 3, 423-440

DOI: $10.24193 /$ subbmath.2021.3.02

\title{
Different type parameterized inequalities via generalized integral operators with applications
}

\author{
Artion Kashuri and Rozana Liko
}

\begin{abstract}
The authors have proved an identity for a generalized integral operator via differentiable function with parameters. By applying the established identity, the generalized trapezium, midpoint and Simpson type integral inequalities have been discovered. It is pointed out that the results of this research provide integral inequalities for almost all fractional integrals discovered in recent past decades. Various special cases have been identified. Some applications of presented results to special means and new error estimates for the trapezium and midpoint quadrature formula have been analyzed. The ideas and techniques of this paper may stimulate further research in the field of integral inequalities.
\end{abstract}

Mathematics Subject Classification (2010): 26A51, 26A33, 26D07, 26D10, 26D15.

Keywords: Trapezium inequality, Simpson inequality, preinvexity, general fractional integrals.

\section{Introduction}

The following inequality, named Hermite-Hadamard inequality, is one of the most famous inequalities in the literature for convex functions.

Theorem 1.1. Let $f: I \subseteq \mathbb{R} \longrightarrow \mathbb{R}$ be a convex function and $e_{1}, e_{2} \in I$ with $e_{1}<e_{2}$. Then the following inequality holds:

$$
f\left(\frac{e_{1}+e_{2}}{2}\right) \leq \frac{1}{e_{2}-e_{1}} \int_{e_{1}}^{e_{2}} f(x) d x \leq \frac{f\left(e_{1}\right)+f\left(e_{2}\right)}{2} .
$$

This inequality (1.1) is also known as trapezium inequality.

The trapezium inequality has remained an area of great interest due to its wide applications in the field of mathematical analysis. Authors of recent decades have studied (1.1) in the premises of newly invented definitions due to motivation of convex function. Interested readers see the references [1]-[10], [12]-[17], [19]-[25].

The following inequality is well known in the literature as Simpson's inequality. 
Theorem 1.2. Let $f:\left[e_{1}, e_{2}\right] \longrightarrow \mathbb{R}$ be four time differentiable on the interval $\left(e_{1}, e_{2}\right)$ and having the fourth derivative bounded on $\left(e_{1}, e_{2}\right)$, that is

$$
\left\|f^{(4)}\right\|_{\infty}=\sup _{x \in\left(e_{1}, e_{2}\right)}\left|f^{(4)}\right|<\infty .
$$

Then, we have

$$
\begin{gathered}
\left|\int_{e_{1}}^{e_{2}} f(x) d x-\frac{e_{2}-e_{1}}{3}\left[\frac{f\left(e_{1}\right)+f\left(e_{2}\right)}{2}+2 f\left(\frac{e_{1}+e_{2}}{2}\right)\right]\right| \\
\leq \frac{1}{2880}\left\|f^{(4)}\right\|_{\infty}\left(e_{2}-e_{1}\right)^{5} .
\end{gathered}
$$

Inequality (1.2) gives an error bound for the classical Simpson quadrature formula, which is one of the most used quadrature formulae in practical applications. In recent years, various generalizations, extensions and variants of such inequalities have been obtained. For other recent results concerning Simpson type inequalities, see [11],[18]. The aim of this paper is to establish trapezium, midpoint and Simpson type generalized integral inequalities for preinvex functions and some new error bounds for midpoint and trapezium quadrature formula. Interestingly, the special cases of presented results, are fractional integral inequalities. Therefore, it is important to summarize the study of fractional integrals.

Let us recall some special functions and evoke some basic definitions as follows:

Definition 1.3. For $k \in \mathbb{R}^{+}$and $x \in \mathbb{C}$, the $k$-gamma function is defined by

$$
\Gamma_{k}(x)=\lim _{n \longrightarrow \infty} \frac{n ! k^{n}(n k)^{\frac{x}{k}-1}}{(x)_{n, k}} .
$$

Its integral representation is given by

$$
\Gamma_{k}(\alpha)=\int_{0}^{\infty} t^{\alpha-1} e^{-\frac{t^{k}}{k}} d t
$$

One can note that

$$
\Gamma_{k}(\alpha+k)=\alpha \Gamma_{k}(\alpha)
$$

For $k=1$, (1.4) gives integral representation of gamma function.

Definition 1.4. [15] Let $f \in L\left[e_{1}, e_{2}\right]$. Then $k$-fractional integrals of order $\alpha, k>0$ with $e_{1} \geq 0$ are defined by

$$
I_{e_{1}^{+}}^{\alpha, k} f(x)=\frac{1}{k \Gamma_{k}(\alpha)} \int_{e_{1}}^{x}(x-t)^{\frac{\alpha}{k}-1} f(t) d t, \quad x>e_{1}
$$

and

$$
I_{e_{2}^{-}}^{\alpha, k} f(x)=\frac{1}{k \Gamma_{k}(\alpha)} \int_{x}^{e_{2}}(t-x)^{\frac{\alpha}{k}-1} f(t) d t, \quad e_{2}>x .
$$

For $k=1, k$-fractional integrals give Riemann-Liouville integrals. For $\alpha=k=1$, $k$-fractional integrals give classical integrals. 
Also, let define a function $\varphi:[0, \infty) \longrightarrow[0, \infty)$ satisfying the following conditions:

$$
\begin{aligned}
& \int_{0}^{1} \frac{\varphi(t)}{t} d t<\infty, \\
& \frac{1}{A} \leq \frac{\varphi(s)}{\varphi(r)} \leq A \text { for } \frac{1}{2} \leq \frac{s}{r} \leq 2 \\
& \frac{\varphi(r)}{r^{2}} \leq B \frac{\varphi(s)}{s^{2}} \text { for } s \leq r \\
& \left|\frac{\varphi(r)}{r^{2}}-\frac{\varphi(s)}{s^{2}}\right| \leq C|r-s| \frac{\varphi(r)}{r^{2}} \text { for } \frac{1}{2} \leq \frac{s}{r} \leq 2
\end{aligned}
$$

where $A, B, C>0$ are independent of $r, s>0$. If $\varphi(r) r^{\alpha}$ is increasing for some $\alpha \geq 0$ and $\frac{\varphi(r)}{r^{\beta}}$ is decreasing for some $\beta \geq 0$, then $\varphi$ satisfies (1.7)-(1.10), see [20]. Therefore, the left-sided and right-sided generalized integral operators are defined as follows:

$$
\begin{gathered}
e_{1}^{+} I_{\varphi} f(x)=\int_{e_{1}}^{x} \frac{\varphi(x-t)}{x-t} f(t) d t, \quad x>e_{1}, \\
e_{2}^{-} I_{\varphi} f(x)=\int_{x}^{e_{2}} \frac{\varphi(t-x)}{t-x} f(t) d t, \quad x<e_{2} .
\end{gathered}
$$

The most important feature of generalized integrals is that; they produce RiemannLiouville fractional integrals, $k$-Riemann-Liouville fractional integrals, Katugampola fractional integrals, conformable fractional integrals, Hadamard fractional integrals, etc., see [19].

Motivated by the above literatures, the main objective of this paper is to discover in Section 2, an interesting identity in order to study some new bounds regarding trapezium, midpoint and Simpson type integral inequalities. By using the established identity as an auxiliary result, some new estimates for trapezium, midpoint and Simpson type integral inequalities via generalized integrals are obtained. It is pointed out that some new fractional integral inequalities have been deduced from main results. In Section 3, some applications to special means and new error estimates for the midpoint and trapezium quadrature formula are given. The ideas and techniques of this paper may stimulate further research in the field of integral inequalities.

\section{Main results}

Throughout this study, let $P=\left[m e_{1}, m e_{1}+\eta\left(e_{2}, m e_{1}\right)\right]$ with $e_{1}<e_{2}, m \in(0,1]$ be an invex subset with respect to $\eta: P \times P \longrightarrow \mathbb{R}$. Also, for brevity, we define

$$
\Lambda_{m}(t):=\int_{0}^{t} \frac{\varphi\left(\eta\left(e_{2}, m e_{1}\right) u\right)}{u} d u<\infty, \quad \eta\left(e_{2}, m e_{1}\right)>0 .
$$

For establishing some new results regarding general fractional integrals we need to prove the following lemma. 
Lemma 2.1. Let $f: P \longrightarrow \mathbb{R}$ be a differentiable mapping on $P^{\circ}$ and $\gamma_{1}, \gamma_{2} \in \mathbb{R}$. If $f^{\prime} \in L(P)$ and $\lambda \in(0,1]$, then the following identity for generalized fractional integrals hold:

$$
\begin{aligned}
& \frac{\gamma_{1} f\left(m e_{1}\right)+\gamma_{2} f\left(m e_{1}+\lambda \eta\left(e_{2}, m e_{1}\right)\right)}{2} \\
& +\left[\frac{2 \lambda \Lambda_{m}\left(\frac{\lambda}{2}\right)}{\eta\left(e_{2}, m e_{1}\right)}-\frac{\gamma_{1}+\gamma_{2}}{2}\right] f\left(m e_{1}+\frac{\lambda}{2} \eta\left(e_{2}, m e_{1}\right)\right)-\frac{\lambda}{\eta\left(e_{2}, m e_{1}\right)} \\
& \times\left[\left(m e_{1}+\frac{\lambda}{2} \eta\left(e_{2}, m e_{1}\right)\right)^{+} I_{\varphi} f\left(m e_{1}+\lambda \eta\left(e_{2}, m e_{1}\right)\right){ }_{\left(m e_{1}+\frac{\lambda}{2} \eta\left(e_{2}, m e_{1}\right)\right)^{-}} I_{\varphi} f\left(m e_{1}\right)\right] \\
& =\frac{\lambda \eta\left(e_{2}, m e_{1}\right)}{2} \\
& \times\left\{\int_{0}^{\frac{1}{2}}\left(\frac{2 \lambda \Lambda_{m}(\lambda t)}{\eta\left(e_{2}, m e_{1}\right)}-\gamma_{1}\right) f^{\prime}\left(m e_{1}+(\lambda t) \eta\left(e_{2}, m e_{1}\right)\right) d t\right. \\
& \left.-\int_{\frac{1}{2}}^{1}\left(\frac{2 \lambda \Lambda_{m}((1-t) \lambda)}{\eta\left(e_{2}, m e_{1}\right)}-\gamma_{2}\right) f^{\prime}\left(m e_{1}+(\lambda t) \eta\left(e_{2}, m e_{1}\right)\right) d t\right\} \text {. }
\end{aligned}
$$

We denote

$$
\begin{gathered}
T_{f, \Lambda_{m}}\left(\lambda, \gamma_{1}, \gamma_{2} ; e_{1}, e_{2}\right):=\frac{\lambda \eta\left(e_{2}, m e_{1}\right)}{2} \\
\times\left\{\int_{0}^{\frac{1}{2}}\left(\frac{2 \lambda \Lambda_{m}(\lambda t)}{\eta\left(e_{2}, m e_{1}\right)}-\gamma_{1}\right) f^{\prime}\left(m e_{1}+(\lambda t) \eta\left(e_{2}, m e_{1}\right)\right) d t\right. \\
\left.-\int_{\frac{1}{2}}^{1}\left(\frac{2 \lambda \Lambda_{m}((1-t) \lambda)}{\eta\left(e_{2}, m e_{1}\right)}-\gamma_{2}\right) f^{\prime}\left(m e_{1}+(\lambda t) \eta\left(e_{2}, m e_{1}\right)\right) d t\right\} .
\end{gathered}
$$

Proof. Integrating by parts eq. (2.3) and changing the variable of integration, we have

$$
\begin{gathered}
T_{f, \Lambda_{m}}\left(\lambda, \gamma_{1}, \gamma_{2} ; e_{1}, e_{2}\right)=\frac{\lambda \eta\left(e_{2}, m e_{1}\right)}{2} \\
\times\left\{\frac{2 \lambda}{\eta\left(e_{2}, m e_{1}\right)} \int_{0}^{\frac{1}{2}} \Lambda_{m}(\lambda t) f^{\prime}\left(m e_{1}+(\lambda t) \eta\left(e_{2}, m e_{1}\right)\right) d t\right. \\
-\gamma_{1} \int_{0}^{\frac{1}{2}} f^{\prime}\left(m e_{1}+(\lambda t) \eta\left(e_{2}, m e_{1}\right)\right) d t \\
-\frac{2 \lambda \quad \gamma_{2}}{\eta\left(e_{2}, m e_{1}\right)} \int_{\frac{1}{2}}^{1} \Lambda_{m}((1-t) \lambda) f^{\prime}\left(m e_{1}+(\lambda t) \eta\left(e_{2}, m e_{1}\right)\right) d t \\
=\frac{\lambda \eta\left(e_{2}, m e_{1}\right)}{2} \times\left\{\left.\frac{2 \Lambda_{m}(\lambda t) f\left(m e_{1}+(\lambda t) \eta\left(e_{2}, m e_{1}\right)\right)}{\eta^{2}\left(e_{2}, m e_{1}\right)}\right|_{0} ^{\frac{1}{2}}\right. \\
-\frac{2 \lambda}{\eta^{2}\left(e_{2}, m e_{1}\right)} \times \int_{0}^{\frac{1}{2}} \frac{\varphi\left(\eta\left(e_{2}, m e_{1}\right)(\lambda t)\right)}{\lambda t} f\left(m e_{1}+(\lambda t) \eta\left(e_{2}, m e_{1}\right)\right) d t
\end{gathered}
$$




$$
\begin{gathered}
-\left.\frac{\gamma_{1}}{\lambda \eta\left(e_{2}, m e_{1}\right)} f\left(m e_{1}+(\lambda t) \eta\left(e_{2}, m e_{1}\right)\right)\right|_{0} ^{\frac{1}{2}} \\
-\left.\frac{2 \Lambda_{m}((1-t) \lambda) f\left(m e_{1}+(\lambda t) \eta\left(e_{2}, m e_{1}\right)\right)}{\eta^{2}\left(e_{2}, m e_{1}\right)}\right|_{\frac{1}{2}} ^{1} \\
-\frac{2 \lambda}{\eta^{2}\left(e_{2}, m e_{1}\right)} \times \int_{\frac{1}{2}}^{1} \frac{\varphi\left(\eta\left(e_{2}, m e_{1}\right)((1-t) \lambda)\right)}{(1-t) \lambda} f\left(m e_{1}+(\lambda t) \eta\left(e_{2}, m e_{1}\right)\right) d t \\
+\left.\frac{\gamma_{2}}{\lambda \eta\left(e_{2}, m e_{1}\right)} f\left(m e_{1}+(\lambda t) \eta\left(e_{2}, m e_{1}\right)\right)\right|_{\frac{1}{2}} ^{1} \\
=\frac{\gamma_{1} f\left(m e_{1}\right)+\gamma_{2} f\left(m e_{1}+\lambda \eta\left(e_{2}, m e_{1}\right)\right)}{2} \\
+\left[\frac{2 \lambda \Lambda_{m}\left(\frac{\lambda}{2}\right)}{\eta\left(e_{2}, m e_{1}\right)}-\frac{\gamma_{1}+\gamma_{2}}{2}\right] f\left(m e_{1}+\frac{\lambda}{2} \eta\left(e_{2}, m e_{1}\right)\right)-\frac{\lambda}{\eta\left(e_{2}, m e_{1}\right)} \\
\times\left[\left(m e_{1}+\frac{\lambda}{2} \eta\left(e_{2}, m e_{1}\right)\right)^{+} I_{\varphi} f\left(m e_{1}+\lambda \eta\left(e_{2}, m e_{1}\right)\right)+\left(m e_{1}+\frac{\lambda}{2} \eta\left(e_{2}, m e_{1}\right)\right)-I_{\varphi} f\left(m e_{1}\right)\right] .
\end{gathered}
$$

This completes the proof of the lemma.

Remark 2.2. a. Taking $\lambda=m=1, \gamma_{1}=\gamma_{2}=0, \eta\left(e_{2}, m e_{1}\right)=e_{2}-m e_{1}$ and $\varphi(t)=t$ in Lemma 2.1, we get the midpoint type identity.

b. Taking $\lambda=m=1, \gamma_{1}=\gamma_{2}=1, \eta\left(e_{2}, m e_{1}\right)=e_{2}-m e_{1}$ and $\varphi(t)=t$ in Lemma 2.1, we get Hermite-Hadamard type identity.

c. Taking $\lambda=m=1, \gamma_{1}=\frac{1}{6}, \gamma_{2}=\frac{5}{6}, \eta\left(e_{2}, m e_{1}\right)=e_{2}-m e_{1}$ and $\varphi(t)=t$ in Lemma 2.1, we get new Simpson type identity.

Theorem 2.3. Let $f: P \longrightarrow \mathbb{R}$ be a differentiable mapping on $P^{\circ}$ and $0 \leq \gamma_{1}, \gamma_{2} \leq 1$. If $\left|f^{\prime}\right|^{q}$ is preinvex on $P$ and $\lambda \in(0,1]$ for $q>1$ and $p^{-1}+q^{-1}=1$, then the following inequality for generalized fractional integrals hold:

$$
\begin{gathered}
\left|T_{f, \Lambda_{m}}\left(\lambda, \gamma_{1}, \gamma_{2} ; e_{1}, e_{2}\right)\right| \leq \frac{\lambda \eta\left(e_{2}, m e_{1}\right)}{2 \sqrt[q]{8}} \\
\times\left\{\sqrt[p]{B_{\Lambda_{m}}\left(\lambda, \gamma_{1} ; p\right)} \times \sqrt[q]{(4-\lambda)\left|f^{\prime}\left(m e_{1}\right)\right|^{q}+\lambda\left|f^{\prime}\left(e_{2}\right)\right|^{q}}\right. \\
\left.+\sqrt[p]{C_{\Lambda_{m}}\left(\lambda, \gamma_{2} ; p\right)} \times \sqrt[q]{(4-3 \lambda)\left|f^{\prime}\left(m e_{1}\right)\right|^{q}+3 \lambda\left|f^{\prime}\left(e_{2}\right)\right|^{q}}\right\},
\end{gathered}
$$

where

$$
B_{\Lambda_{m}}\left(\lambda, \gamma_{1} ; p\right):=\int_{0}^{\frac{1}{2}}\left|\frac{2 \lambda \Lambda_{m}(\lambda t)}{\eta\left(e_{2}, m e_{1}\right)}-\gamma_{1}\right|^{p} d t
$$

and

$$
C_{\Lambda_{m}}\left(\lambda, \gamma_{2} ; p\right):=\int_{\frac{1}{2}}^{1}\left|\frac{2 \lambda \Lambda_{m}((1-t) \lambda)}{\eta\left(e_{2}, m e_{1}\right)}-\gamma_{2}\right|^{p} d t
$$


Proof. From Lemma 2.1, preinvexity of $\left|f^{\prime}\right|^{q}$, Hölder inequality and properties of the modulus, we have

$$
\begin{aligned}
& \left|T_{f, \Lambda_{m}}\left(\lambda, \gamma_{1}, \gamma_{2} ; e_{1}, e_{2}\right)\right| \leq \frac{\lambda \eta\left(e_{2}, m e_{1}\right)}{2} \\
& \times\left\{\int_{0}^{\frac{1}{2}}\left|\frac{2 \lambda \Lambda_{m}(\lambda t)}{\eta\left(e_{2}, m e_{1}\right)}-\gamma_{1}\right|\left|f^{\prime}\left(m e_{1}+(\lambda t) \eta\left(e_{2}, m e_{1}\right)\right)\right| d t\right. \\
& \left.+\int_{\frac{1}{2}}^{1}\left|\frac{2 \lambda \Lambda_{m}((1-t) \lambda)}{\eta\left(e_{2}, m e_{1}\right)}-\gamma_{2}\right|\left|f^{\prime}\left(m e_{1}+(\lambda t) \eta\left(e_{2}, m e_{1}\right)\right)\right| d t\right\} \\
& \leq \frac{\lambda \eta\left(e_{2}, m e_{1}\right)}{2} \\
& \times\left\{\left(\int_{0}^{\frac{1}{2}}\left|\frac{2 \lambda \Lambda_{m}(\lambda t)}{\eta\left(e_{2}, m e_{1}\right)}-\gamma_{1}\right|^{p} d t\right)^{\frac{1}{p}}\left(\int_{0}^{\frac{1}{2}}\left|f^{\prime}\left(m e_{1}+(\lambda t) \eta\left(e_{2}, m e_{1}\right)\right)\right|^{q} d t\right)^{\frac{1}{q}}\right. \\
& \left.+\left(\int_{\frac{1}{2}}^{1}\left|\frac{2 \lambda \Lambda_{m}((1-t) \lambda)}{\eta\left(e_{2}, m e_{1}\right)}-\gamma_{2}\right|^{p} d t\right)^{\frac{1}{p}}\left(\int_{\frac{1}{2}}^{1}\left|f^{\prime}\left(m e_{1}+(\lambda t) \eta\left(e_{2}, m e_{1}\right)\right)\right|^{q} d t\right)^{\frac{1}{q}}\right\} \\
& \leq \frac{\lambda \eta\left(e_{2}, m e_{1}\right)}{2} \times\left\{\sqrt[p]{B_{\Lambda_{m}}\left(\lambda, \gamma_{1} ; p\right)}\right. \\
& \times\left(\int_{0}^{\frac{1}{2}}\left[(1-\lambda t)\left|f^{\prime}\left(m e_{1}\right)\right|^{q}+(\lambda t)\left|f^{\prime}\left(e_{2}\right)\right|^{q}\right] d t\right)^{\frac{1}{q}} \\
& +\sqrt[p]{C_{\Lambda_{m}}\left(\lambda, \gamma_{2} ; p\right)} \\
& \left.\times\left(\int_{\frac{1}{2}}^{1}\left[(1-\lambda t)\left|f^{\prime}\left(m e_{1}\right)\right|^{q}+(\lambda t)\left|f^{\prime}\left(e_{2}\right)\right|^{q}\right] d t\right)^{\frac{1}{q}}\right\} \\
& =\frac{\lambda \eta\left(e_{2}, m e_{1}\right)}{2 \sqrt[q]{8}} \\
& \times\left\{\sqrt[p]{B_{\Lambda_{m}}\left(\lambda, \gamma_{1} ; p\right)} \times \sqrt[q]{(4-\lambda)\left|f^{\prime}\left(m e_{1}\right)\right|^{q}+\lambda\left|f^{\prime}\left(e_{2}\right)\right|^{q}}\right. \\
& \left.+\sqrt[p]{C_{\Lambda_{m}}\left(\lambda, \gamma_{2} ; p\right)} \times \sqrt[q]{(4-3 \lambda)\left|f^{\prime}\left(m e_{1}\right)\right|^{q}+3 \lambda\left|f^{\prime}\left(e_{2}\right)\right|^{q}}\right\} .
\end{aligned}
$$

The proof of this theorem is complete.

We point out some special cases of Theorem 2.3 .

Corollary 2.4. Taking $p=q=2$ in Theorem 2.3, we get

$$
\begin{gathered}
\left|T_{f, \Lambda_{m}}\left(\lambda, \gamma_{1}, \gamma_{2} ; e_{1}, e_{2}\right)\right| \leq \frac{\lambda \eta\left(e_{2}, m e_{1}\right)}{4 \sqrt{2}} \\
\times\left\{\sqrt{B_{\Lambda_{m}}\left(\lambda, \gamma_{1} ; 2\right)} \times \sqrt{(4-\lambda)\left|f^{\prime}\left(m e_{1}\right)\right|^{2}+\lambda\left|f^{\prime}\left(e_{2}\right)\right|^{2}}\right. \\
\left.+\sqrt{C_{\Lambda_{m}}\left(\lambda, \gamma_{2} ; 2\right)} \times \sqrt{(4-3 \lambda)\left|f^{\prime}\left(m e_{1}\right)\right|^{2}+3 \lambda\left|f^{\prime}\left(e_{2}\right)\right|^{2}}\right\} .
\end{gathered}
$$


Corollary 2.5. Taking $\left|f^{\prime}\right| \leq K$ in Theorem 2.3, we get

$$
\begin{aligned}
& \left|T_{f, \Lambda_{m}}\left(\lambda, \gamma_{1}, \gamma_{2} ; e_{1}, e_{2}\right)\right| \leq \frac{K \lambda \eta\left(e_{2}, m e_{1}\right)}{2 \sqrt[q]{2}} \\
& \times\left\{\sqrt[p]{B_{\Lambda_{m}}\left(\lambda, \gamma_{1} ; p\right)}+\sqrt[p]{C_{\Lambda_{m}}\left(\lambda, \gamma_{2} ; p\right)}\right\} .
\end{aligned}
$$

Corollary 2.6. Taking $\lambda=m=1, \gamma_{1}=\gamma_{2}=0, \eta\left(e_{2}, m e_{1}\right)=e_{2}-m e_{1}$ and $\varphi(t)=t$ in Theorem 2.3, we get the following midpoint type inequality:

$$
\begin{gathered}
\left|T_{f}\left(1,0,0 ; e_{1}, e_{2}\right)\right| \leq \frac{\sqrt[p]{2}\left(e_{2}-e_{1}\right)}{2 \sqrt[q]{8} \sqrt[p]{2^{p+1}(p+1)}} \\
\times\left\{\sqrt[q]{\left|f^{\prime}\left(e_{1}\right)\right|^{q}+3\left|f^{\prime}\left(e_{2}\right)\right|^{q}}+\sqrt[q]{3\left|f^{\prime}\left(e_{1}\right)\right|^{q}+\left|f^{\prime}\left(e_{2}\right)\right|^{q}}\right\} .
\end{gathered}
$$

Corollary 2.7. Taking $\lambda=m=1, \gamma_{1}=\gamma_{2}=1, \eta\left(e_{2}, m e_{1}\right)=e_{2}-m e_{1}$ and $\varphi(t)=t$ in Theorem 2.3, we get the following trapezium type inequality:

$$
\begin{gathered}
\left|T_{f}\left(1,1,1 ; e_{1}, e_{2}\right)\right| \leq \frac{\left(e_{2}-e_{1}\right)}{2 \sqrt[q]{8} \sqrt[p]{2(p+1)}} \\
\times\left\{\sqrt[q]{\left|f^{\prime}\left(e_{1}\right)\right|^{q}+3\left|f^{\prime}\left(e_{2}\right)\right|^{q}}+\sqrt[q]{3\left|f^{\prime}\left(e_{1}\right)\right|^{q}+\left|f^{\prime}\left(e_{2}\right)\right|^{q}}\right\} .
\end{gathered}
$$

Corollary 2.8. Taking $\lambda=m=1, \gamma_{1}=\frac{1}{6}, \gamma_{2}=\frac{5}{6}, \eta\left(e_{2}, m e_{1}\right)=e_{2}-m e_{1}$ and $\varphi(t)=t$ in Theorem 2.3, we get the following Simpson type inequality:

$$
\begin{gathered}
\left|T_{f}\left(1, \frac{1}{6}, \frac{5}{6} ; e_{1}, e_{2}\right)\right| \leq \frac{\sqrt[p]{5^{p+1}+1}\left(e_{2}-e_{1}\right)}{12 \sqrt[q]{8} \sqrt[p]{12(p+1)}} \\
\times\left\{\sqrt[q]{\left|f^{\prime}\left(e_{1}\right)\right|^{q}+3\left|f^{\prime}\left(e_{2}\right)\right|^{q}}+\sqrt[q]{3\left|f^{\prime}\left(e_{1}\right)\right|^{q}+\left|f^{\prime}\left(e_{2}\right)\right|^{q}}\right\} .
\end{gathered}
$$

Theorem 2.9. Let $f: P \longrightarrow \mathbb{R}$ be a differentiable mapping on $P^{\circ}$ and $0 \leq \gamma_{1}, \gamma_{2} \leq 1$. If $\left|f^{\prime}\right|^{q}$ is preinvex on $P$ and $\lambda \in(0,1]$ for $q \geq 1$, then the following inequality for generalized fractional integrals hold:

$$
\begin{gathered}
\left|T_{f, \Lambda_{m}}\left(\lambda, \gamma_{1}, \gamma_{2} ; e_{1}, e_{2}\right)\right| \leq \frac{\lambda \eta\left(e_{2}, m e_{1}\right)}{2} \times\left\{\left[B_{\Lambda_{m}}\left(\lambda, \gamma_{1} ; 1\right)\right]^{1-\frac{1}{q}}\right. \\
\times \sqrt[q]{\left[B_{\Lambda_{m}}\left(\lambda, \gamma_{1} ; 1\right)-\lambda D_{\Lambda_{m}}\left(\lambda, \gamma_{1}\right)\right]\left|f^{\prime}\left(m e_{1}\right)\right|^{q}+\lambda D_{\Lambda_{m}}\left(\lambda, \gamma_{1}\right)\left|f^{\prime}\left(e_{2}\right)\right|^{q}} \\
+\left[C_{\Lambda_{m}}\left(\lambda, \gamma_{2} ; 1\right)\right]^{1-\frac{1}{q}} \\
\left.\times \sqrt[q]{\left[C_{\Lambda_{m}}\left(\lambda, \gamma_{2} ; 1\right)-\lambda E_{\Lambda_{m}}\left(\lambda, \gamma_{2}\right)\right]\left|f^{\prime}\left(m e_{1}\right)\right|^{q}+\lambda E_{\Lambda_{m}}\left(\lambda, \gamma_{2}\right)\left|f^{\prime}\left(e_{2}\right)\right|^{q}}\right\}
\end{gathered}
$$

where

$$
D_{\Lambda_{m}}\left(\lambda, \gamma_{1}\right):=\int_{0}^{\frac{1}{2}} t\left|\frac{2 \lambda \Lambda_{m}(\lambda t)}{\eta\left(e_{2}, m e_{1}\right)}-\gamma_{1}\right| d t
$$




$$
E_{\Lambda_{m}}\left(\lambda, \gamma_{2}\right):=\int_{\frac{1}{2}}^{1} t\left|\frac{2 \lambda \Lambda_{m}((1-t) \lambda)}{\eta\left(e_{2}, m e_{1}\right)}-\gamma_{2}\right| d t
$$

and $B_{\Lambda_{m}}\left(\lambda, \gamma_{1} ; 1\right), C_{\Lambda_{m}}\left(\lambda, \gamma_{2} ; 1\right)$ are defined as in Theorem 2.3.

Proof. From Lemma 2.1, preinvexity of $\left|f^{\prime}\right|^{q}$, power mean inequality and properties of the modulus, we have

$$
\begin{aligned}
& \left|T_{f, \Lambda_{m}}\left(\lambda, \gamma_{1}, \gamma_{2} ; e_{1}, e_{2}\right)\right| \leq \frac{\lambda \eta\left(e_{2}, m e_{1}\right)}{2} \\
& \times\left\{\int_{0}^{\frac{1}{2}}\left|\frac{2 \lambda \Lambda_{m}(\lambda t)}{\eta\left(e_{2}, m e_{1}\right)}-\gamma_{1}\right|\left|f^{\prime}\left(m e_{1}+(\lambda t) \eta\left(e_{2}, m e_{1}\right)\right)\right| d t\right. \\
& \left.+\int_{\frac{1}{2}}^{1}\left|\frac{2 \lambda \Lambda_{m}((1-t) \lambda)}{\eta\left(e_{2}, m e_{1}\right)}-\gamma_{2}\right|\left|f^{\prime}\left(m e_{1}+(\lambda t) \eta\left(e_{2}, m e_{1}\right)\right)\right| d t\right\} \\
& \leq \frac{\lambda \eta\left(e_{2}, m e_{1}\right)}{2} \times\left\{\left(\int_{0}^{\frac{1}{2}}\left|\frac{2 \lambda \Lambda_{m}(\lambda t)}{\eta\left(e_{2}, m e_{1}\right)}-\gamma_{1}\right| d t\right)^{1-\frac{1}{q}}\right. \\
& \times\left(\int_{0}^{\frac{1}{2}}\left|\frac{2 \lambda \Lambda_{m}(\lambda t)}{\eta\left(e_{2}, m e_{1}\right)}-\gamma_{1}\right|\left|f^{\prime}\left(m e_{1}+(\lambda t) \eta\left(e_{2}, m e_{1}\right)\right)\right|^{q} d t\right)^{\frac{1}{q}} \\
& +\left(\int_{\frac{1}{2}}^{1}\left|\frac{2 \lambda \Lambda_{m}((1-t) \lambda)}{\eta\left(e_{2}, m e_{1}\right)}-\gamma_{2}\right| d t\right)^{1-\frac{1}{q}} \\
& \left.\times\left(\int_{\frac{1}{2}}^{1}\left|\frac{2 \lambda \Lambda_{m}((1-t) \lambda)}{\eta\left(e_{2}, m e_{1}\right)}-\gamma_{2}\right|\left|f^{\prime}\left(m e_{1}+(\lambda t) \eta\left(e_{2}, m e_{1}\right)\right)\right|^{q} d t\right)^{\frac{1}{q}}\right\} \\
& \leq \frac{\lambda \eta\left(e_{2}, m e_{1}\right)}{2} \times\left[B_{\Lambda_{m}}\left(\lambda, \gamma_{1} ; 1\right)\right]^{1-\frac{1}{q}} \\
& \times\left\{\left(\int_{0}^{\frac{1}{2}}\left|\frac{2 \lambda \Lambda_{m}(\lambda t)}{\eta\left(e_{2}, m e_{1}\right)}-\gamma_{1}\right|\left[(1-\lambda t)\left|f^{\prime}\left(m e_{1}\right)\right|^{q}+(\lambda t)\left|f^{\prime}\left(e_{2}\right)\right|^{q}\right] d t\right)^{\frac{1}{q}}\right. \\
& +\left[C_{\Lambda_{m}}\left(\lambda, \gamma_{2} ; 1\right)\right]^{1-\frac{1}{q}} \\
& \times\left\{\left(\int_{\frac{1}{2}}^{1}\left|\frac{2 \lambda \Lambda_{m}((1-t) \lambda)}{\eta\left(e_{2}, m e_{1}\right)}-\gamma_{2}\right|\left[(1-\lambda t)\left|f^{\prime}\left(m e_{1}\right)\right|^{q}+(\lambda t)\left|f^{\prime}\left(e_{2}\right)\right|^{q}\right] d t\right)^{\frac{1}{q}}\right\} \\
& =\frac{\lambda \eta\left(e_{2}, m e_{1}\right)}{2} \times\left\{\left[B_{\Lambda_{m}}\left(\lambda, \gamma_{1} ; 1\right)\right]^{1-\frac{1}{q}}\right. \\
& \times \sqrt[q]{\left[B_{\Lambda_{m}}\left(\lambda, \gamma_{1} ; 1\right)-\lambda D_{\Lambda_{m}}\left(\lambda, \gamma_{1}\right)\right]\left|f^{\prime}\left(m e_{1}\right)\right|^{q}+\lambda D_{\Lambda_{m}}\left(\lambda, \gamma_{1}\right)\left|f^{\prime}\left(e_{2}\right)\right|^{q}} \\
& +\left[C_{\Lambda_{m}}\left(\lambda, \gamma_{2} ; 1\right)\right]^{1-\frac{1}{q}}
\end{aligned}
$$




$$
\left.\times \sqrt[q]{\left[C_{\Lambda_{m}}\left(\lambda, \gamma_{2} ; 1\right)-\lambda E_{\Lambda_{m}}\left(\lambda, \gamma_{2}\right)\right]\left|f^{\prime}\left(m e_{1}\right)\right|^{q}+\lambda E_{\Lambda_{m}}\left(\lambda, \gamma_{2}\right)\left|f^{\prime}\left(e_{2}\right)\right|^{q}}\right\}
$$

The proof of this theorem is complete.

We point out some special cases of Theorem 2.9 .

Corollary 2.10. Taking $q=1$ in Theorem 2.9, we get

$$
\begin{gathered}
\left|T_{f, \Lambda_{m}}\left(\lambda, \gamma_{1}, \gamma_{2} ; e_{1}, e_{2}\right)\right| \leq \frac{\lambda \eta\left(e_{2}, m e_{1}\right)}{2} \\
\times\left\{\left[B_{\Lambda_{m}}\left(\lambda, \gamma_{1} ; 1\right)-\lambda D_{\Lambda_{m}}\left(\lambda, \gamma_{1}\right)\right]\left|f^{\prime}\left(m e_{1}\right)\right|+\lambda D_{\Lambda_{m}}\left(\lambda, \gamma_{1}\right)\left|f^{\prime}\left(e_{2}\right)\right|\right. \\
\left.+\left[C_{\Lambda_{m}}\left(\lambda, \gamma_{2} ; 1\right)-\lambda E_{\Lambda_{m}}\left(\lambda, \gamma_{2}\right)\right]\left|f^{\prime}\left(m e_{1}\right)\right|+\lambda E_{\Lambda_{m}}\left(\lambda, \gamma_{2}\right)\left|f^{\prime}\left(e_{2}\right)\right|\right\} .
\end{gathered}
$$

Corollary 2.11. Taking $\left|f^{\prime}\right| \leq K$ in Theorem 2.9, we get

$$
\begin{gathered}
\left|T_{f, \Lambda_{m}}\left(\lambda, \gamma_{1}, \gamma_{2} ; e_{1}, e_{2}\right)\right| \leq \frac{K \lambda \eta\left(e_{2}, m e_{1}\right)}{2} \\
\quad \times\left\{B_{\Lambda_{m}}\left(\lambda, \gamma_{1} ; 1\right)+C_{\Lambda_{m}}\left(\lambda, \gamma_{2} ; 1\right)\right\} .
\end{gathered}
$$

Corollary 2.12. Taking $\lambda=m=1, \gamma_{1}=\gamma_{2}=0, \eta\left(e_{2}, m e_{1}\right)=e_{2}-m e_{1}$ and $\varphi(t)=t$ in Theorem 2.9, we get the following midpoint type inequality:

$$
\begin{gathered}
\left|T_{f}\left(1,0,0 ; e_{1}, e_{2}\right)\right| \leq \frac{\left(e_{2}-e_{1}\right)}{8 \sqrt[q]{3}} \\
\times\left\{\sqrt[q]{\left|f^{\prime}\left(e_{1}\right)\right|^{q}+2\left|f^{\prime}\left(e_{2}\right)\right|^{q}}+\sqrt[q]{2\left|f^{\prime}\left(e_{1}\right)\right|^{q}+\left|f^{\prime}\left(e_{2}\right)\right|^{q}}\right\} .
\end{gathered}
$$

Corollary 2.13. Taking $\lambda=m=1, \gamma_{1}=\gamma_{2}=1, \eta\left(e_{2}, m e_{1}\right)=e_{2}-m e_{1}$ and $\varphi(t)=t$ in Theorem 2.9, we get the following trapezium type inequality:

$$
\begin{gathered}
\left|T_{f}\left(1,1,1 ; e_{1}, e_{2}\right)\right| \leq \frac{\left(e_{2}-e_{1}\right)}{8 \sqrt[q]{6}} \\
\times\left\{\sqrt[q]{\left|f^{\prime}\left(e_{1}\right)\right|^{q}+5\left|f^{\prime}\left(e_{2}\right)\right|^{q}}+\sqrt[q]{5\left|f^{\prime}\left(e_{1}\right)\right|^{q}+\left|f^{\prime}\left(e_{2}\right)\right|^{q}}\right\} .
\end{gathered}
$$

Corollary 2.14. Taking $\lambda=m=1, \gamma_{1}=\frac{1}{6}, \gamma_{2}=\frac{5}{6}, \eta\left(e_{2}, m e_{1}\right)=e_{2}-m e_{1}$ and $\varphi(t)=t$ in Theorem 2.9, we get the following Simpson type inequality:

$$
\begin{gathered}
\left|T_{f, \Lambda_{1}}\left(1, \frac{1}{6}, \frac{5}{6} ; e_{1}, e_{2}\right)\right| \leq\left(\frac{13}{72}\right)^{1-\frac{1}{q}} \frac{\left(e_{2}-e_{1}\right)}{2} \\
\times\left\{\frac{\sqrt[q]{305\left|f^{\prime}\left(e_{1}\right)\right|^{q}+163\left|f^{\prime}\left(e_{2}\right)\right|^{q}}}{\sqrt[q]{2592}}+\frac{\sqrt[q]{5938\left|f^{\prime}\left(e_{1}\right)\right|^{q}+1550\left|f^{\prime}\left(e_{2}\right)\right|^{q}}}{\sqrt[q]{41472}}\right\} .
\end{gathered}
$$


Remark 2.15. Applying our Theorems 2.3 and 2.9 for special values of parameter $\lambda, \gamma_{1}$ and $\gamma_{2}$, for appropriate choices of function

$$
\varphi(t)=\frac{t^{\alpha}}{\Gamma(\alpha)}, \frac{t^{\frac{\alpha}{k}}}{k \Gamma_{k}(\alpha)} ; \quad \varphi(t)=t\left(e_{2}-t\right)^{\alpha-1}
$$

for $\alpha \in(0,1) ; \varphi(t)=\frac{t}{\alpha} \exp \left[\left(-\frac{1-\alpha}{\alpha}\right) t\right]$ for $\alpha \in(0,1)$, such that $\left|f^{\prime}\right|^{q}$ to be preinvex (or convex in special case), we can deduce some new general fractional integral inequalities. The details are left to the interested reader.

\section{Applications}

Consider the following special means for different real numbers $\alpha, \beta$ and $\alpha \beta \neq 0$, as follows.

1. The arithmetic mean:

$$
A:=A(\alpha, \beta)=\frac{\alpha+\beta}{2}
$$

2. The harmonic mean:

$$
H:=H(\alpha, \beta)=\frac{2}{\frac{1}{\alpha}+\frac{1}{\beta}},
$$

3. The logarithmic mean:

$$
L:=L(\alpha, \beta)=\frac{\beta-\alpha}{\ln |\beta|-\ln |\alpha|},
$$

4. The generalized log-mean:

$$
L_{r}:=L_{r}(\alpha, \beta)=\left[\frac{\beta^{r+1}-\alpha^{r+1}}{(r+1)(\beta-\alpha)}\right]^{\frac{1}{r}} ; r \in \mathbb{Z} \backslash\{-1,0\} .
$$

It is well known that $L_{r}$ is monotonic nondecreasing over $r \in \mathbb{Z}$ with $L_{-1}:=L$. In particular, we have the following inequality $H \leq L \leq A$. Now, using the theory results in Section 2, we give some applications to special means for different real numbers.

Proposition 3.1. Let $e_{1}, e_{2} \in \mathbb{R} \backslash\{0\}$, where $e_{1}<e_{2}$. Then for $r \in \mathbb{N}$ and $r \geq 2$, where $q>1$ and $p^{-1}+q^{-1}=1$, the following inequality hold:

$$
\begin{gathered}
\left.\left|A^{r}\left(e_{1}, e_{2}\right)-L_{r}^{r}\left(e_{1}, e_{2}\right)\right| \leq \frac{r\left(e_{2}-e_{1}\right)}{\sqrt[q]{8}{\sqrt[p]{2^{p+1}(p+1)}}^{(j)}}+\sqrt[q]{A\left(3\left|e_{1}\right|^{q(r-1)},\left|e_{2}\right|^{q(r-1)}\right)}\right\} . \\
\times\left\{\sqrt[q]{A\left(\left|e_{1}\right|^{q(r-1)}, 3(r-1)\right.}\right\}
\end{gathered}
$$

Proof. Taking $\lambda=m=1, \gamma_{1}=\gamma_{2}=0, \eta\left(e_{2}, m e_{1}\right)=e_{2}-m e_{1}, f(t)=t^{r}$ and $\varphi(t)=t$, in Theorem 2.3 , one can obtain the result immediately. 
Proposition 3.2. Let $e_{1}, e_{2} \in \mathbb{R} \backslash\{0\}$, where $e_{1}<e_{2}$. Then for $r \in \mathbb{N}$ and $r \geq 2$, where $q>1$ and $p^{-1}+q^{-1}=1$, the following inequality hold:

$$
\begin{gathered}
\left|A\left(e_{1}^{r}, e_{2}^{r}\right)-L_{r}^{r}\left(e_{1}, e_{2}\right)\right| \leq \frac{r\left(e_{2}-e_{1}\right)}{2 \sqrt[q]{4} \sqrt[p]{2(p+1)}} \\
\times\left\{\sqrt[q]{A\left(\left|e_{1}\right|^{q(r-1)}, 3\left|e_{2}\right|^{q(r-1)}\right)}+\sqrt[q]{A\left(3\left|e_{1}\right|^{q(r-1)},\left|e_{2}\right|^{q(r-1)}\right)}\right\} .
\end{gathered}
$$

Proof. Taking $\lambda=m=1, \gamma_{1}=\gamma_{2}=1, \eta\left(e_{2}, m e_{1}\right)=e_{2}-m e_{1}, f(t)=t^{r}$ and $\varphi(t)=t$, in Theorem 2.3 , one can obtain the result immediately.

Proposition 3.3. Let $e_{1}, e_{2} \in \mathbb{R} \backslash\{0\}$, where $e_{1}<e_{2}$. Then for $r \in \mathbb{N}$ and $r \geq 2$, where $q>1$ and $p^{-1}+q^{-1}=1$, the following inequality hold:

$$
\begin{aligned}
& \left|\frac{A\left(e_{1}^{r}, 5 e_{2}^{r}\right)}{6}+\frac{A^{r}\left(e_{1}, e_{2}\right)}{2}-L_{r}^{r}\left(e_{1}, e_{2}\right)\right| \leq \frac{r \sqrt[p]{5^{p+1}+1}\left(e_{2}-e_{1}\right)}{12 \sqrt[q]{4} \sqrt[p]{12(p+1)}} \\
& \times\left\{\sqrt[q]{A\left(\left|e_{1}\right|^{q(r-1)}, 3\left|e_{2}\right|^{q(r-1)}\right)}+\sqrt[q]{A\left(3\left|e_{1}\right|^{q(r-1)},\left|e_{2}\right|^{q(r-1)}\right)}\right\} .
\end{aligned}
$$

Proof. Taking $\lambda=m=1, \gamma_{1}=\frac{1}{6}, \gamma_{2}=\frac{5}{6}, \eta\left(e_{2}, m e_{1}\right)=e_{2}-m e_{1}, f(t)=t^{r}$ and $\varphi(t)=t$, in Theorem 2.3 , one can obtain the result immediately.

Proposition 3.4. Let $e_{1}, e_{2} \in \mathbb{R} \backslash\{0\}$, where $e_{1}<e_{2}$. Then for $q>1$ and $p^{-1}+q^{-1}=1$, the following inequality hold:

$$
\begin{aligned}
& \left|\frac{1}{A\left(e_{1}, e_{2}\right)}-\frac{1}{L\left(e_{1}, e_{2}\right)}\right| \leq \sqrt[q]{\frac{3}{8}} \frac{\left(e_{2}-e_{1}\right)}{\sqrt[p]{2^{p+1}(p+1)}} \\
& \times\left\{\frac{1}{\sqrt[q]{H\left(\left|e_{1}\right|^{2 q}, 3\left|e_{2}\right|^{2 q}\right)}}+\frac{1}{\sqrt[q]{H\left(3\left|e_{1}\right|^{2 q},\left|e_{2}\right|^{2 q}\right)}}\right\} .
\end{aligned}
$$

Proof. Taking $\lambda=m=1, \gamma_{1}=\gamma_{2}=0, \eta\left(e_{2}, m e_{1}\right)=e_{2}-m e_{1}, f(t)=\frac{1}{t}$ and $\varphi(t)=t$, in Theorem 2.3 , one can obtain the result immediately.

Proposition 3.5. Let $e_{1}, e_{2} \in \mathbb{R} \backslash\{0\}$, where $e_{1}<e_{2}$. Then for $q>1$ and $p^{-1}+q^{-1}=1$, the following inequality hold:

$$
\begin{aligned}
&\left|\frac{1}{H\left(e_{1}, e_{2}\right)}-\frac{1}{L\left(e_{1}, e_{2}\right)}\right| \leq \sqrt[q]{\frac{3}{4} \frac{\left(e_{2}-e_{1}\right)}{2 \sqrt[p]{2(p+1)}}} \\
& \times\left\{\frac{1}{\sqrt[q]{H\left(\left|e_{1}\right|^{2 q}, 3\left|e_{2}\right|^{2 q}\right)}}+\frac{1}{\sqrt[q]{H\left(3\left|e_{1}\right|^{2 q},\left|e_{2}\right|^{2 q}\right)}}\right\} .
\end{aligned}
$$

Proof. Taking $\lambda=m=1, \gamma_{1}=\gamma_{2}=1, \eta\left(e_{2}, m e_{1}\right)=e_{2}-m e_{1}, f(t)=\frac{1}{t}$ and $\varphi(t)=t$, in Theorem 2.3 , one can obtain the result immediately. 
Proposition 3.6. Let $e_{1}, e_{2} \in \mathbb{R} \backslash\{0\}$, where $e_{1}<e_{2}$. Then for $q>1$ and $p^{-1}+q^{-1}=1$, the following inequality hold:

$$
\begin{gathered}
\left|\frac{5}{6 H\left(5 e_{1}, e_{2}\right)}+\frac{1}{2 A\left(e_{1}, e_{2}\right)}-\frac{1}{L\left(e_{1}, e_{2}\right)}\right| \leq \sqrt[q]{\frac{3}{4}} \frac{\sqrt[p]{5^{p+1}+1}\left(e_{2}-e_{1}\right)}{12 \sqrt[p]{12(p+1)}} \\
\quad \times\left\{\frac{1}{\sqrt[q]{H\left(\left|e_{1}\right|^{2 q}, 3\left|e_{2}\right|^{2 q}\right)}}+\frac{1}{\sqrt[q]{H\left(3\left|e_{1}\right|^{2 q},\left|e_{2}\right|^{2 q}\right)}}\right\} .
\end{gathered}
$$

Proof. Taking $\lambda=m=1, \gamma_{1}=\frac{1}{5}, \gamma_{2}=\frac{5}{6}, \eta\left(e_{2}, m e_{1}\right)=e_{2}-m e_{1}, f(t)=\frac{1}{t}$ and $\varphi(t)=t$, in Theorem 2.3 , one can obtain the result immediately.

Proposition 3.7. Let $e_{1}, e_{2} \in \mathbb{R} \backslash\{0\}$, where $e_{1}<e_{2}$. Then for $r \in \mathbb{N}$ and $r \geq 2$, where $q \geq 1$, the following inequality hold:

$$
\begin{gathered}
\left|A^{r}\left(e_{1}, e_{2}\right)-L_{r}^{r}\left(e_{1}, e_{2}\right)\right| \leq \sqrt[q]{\frac{2}{3}} \frac{r\left(e_{2}-e_{1}\right)}{8} \\
\times\left\{\sqrt[q]{A\left(\left|e_{1}\right|^{q(r-1)}, 2\left|e_{2}\right|^{q(r-1)}\right)}+\sqrt[q]{A\left(2\left|e_{1}\right|^{q(r-1)},\left|e_{2}\right|^{q(r-1)}\right)}\right\} .
\end{gathered}
$$

Proof. Taking $\lambda=m=1, \gamma_{1}=\gamma_{2}=0, \eta\left(e_{2}, m e_{1}\right)=e_{2}-m e_{1}, f(t)=t^{r}$ and $\varphi(t)=t$, in Theorem 2.9, one can obtain the result immediately.

Proposition 3.8. Let $e_{1}, e_{2} \in \mathbb{R} \backslash\{0\}$, where $e_{1}<e_{2}$. Then for $r \in \mathbb{N}$ and $r \geq 2$, where $q \geq 1$, the following inequality hold:

$$
\begin{gathered}
\left|A\left(e_{1}^{r}, e_{2}^{r}\right)-L_{r}^{r}\left(e_{1}, e_{2}\right)\right| \leq \frac{r\left(e_{2}-e_{1}\right)}{8 \sqrt[q]{3}} \\
\times\left\{\sqrt[q]{A\left(\left|e_{1}\right|^{q(r-1)}, 5\left|e_{2}\right|^{q(r-1)}\right)}+\sqrt[q]{A\left(5\left|e_{1}\right|^{q(r-1)},\left|e_{2}\right|^{q(r-1)}\right)}\right\} .
\end{gathered}
$$

Proof. Taking $\lambda=m=1, \gamma_{1}=\gamma_{2}=1, \eta\left(e_{2}, m e_{1}\right)=e_{2}-m e_{1}, f(t)=t^{r}$ and $\varphi(t)=t$, in Theorem 2.9, one can obtain the result immediately.

Proposition 3.9. Let $e_{1}, e_{2} \in \mathbb{R} \backslash\{0\}$, where $e_{1}<e_{2}$. Then for $r \in \mathbb{N}$ and $r \geq 2$, where $q \geq 1$, the following inequality hold:

$$
\begin{gathered}
\left|\frac{A\left(e_{1}^{r}, 5 e_{2}^{r}\right)}{6}+\frac{A^{r}\left(e_{1}, e_{2}\right)}{2}-L_{r}^{r}\left(e_{1}, e_{2}\right)\right| \leq\left(\frac{13}{72}\right)^{1-\frac{1}{q}} \frac{r\left(e_{2}-e_{1}\right)}{2} \\
\times\left\{\frac{\left.\sqrt[q]{A\left(305\left|e_{1}\right| q(r-1)\right.}, 163\left|e_{2}\right|^{q(r-1)}\right)}{\sqrt[q]{1296}}+\frac{\sqrt[q]{A\left(5938\left|e_{1}\right|^{q(r-1)}, 1550\left|e_{2}\right|^{q(r-1)}\right)}}{\sqrt[q]{20736}}\right\} .
\end{gathered}
$$

Proof. Taking $\lambda=m=1, \gamma_{1}=\frac{1}{6}, \gamma_{2}=\frac{5}{6}, \eta\left(e_{2}, m e_{1}\right)=e_{2}-m e_{1}, f(t)=t^{r}$ and $\varphi(t)=t$, in Theorem 2.9, one can obtain the result immediately. 
Proposition 3.10. Let $e_{1}, e_{2} \in \mathbb{R} \backslash\{0\}$, where $e_{1}<e_{2}$. Then for $q \geq 1$, the following inequality hold:

$$
\begin{gathered}
\left|\frac{1}{A\left(e_{1}, e_{2}\right)}-\frac{1}{L\left(e_{1}, e_{2}\right)}\right| \leq \sqrt[q]{\frac{4}{3} \frac{\left(e_{2}-e_{1}\right)}{8}} \\
\times\left\{\frac{1}{\sqrt[q]{H\left(\left|e_{1}\right|^{2 q}, 2\left|e_{2}\right|^{2 q}\right)}}+\frac{1}{\sqrt[q]{H\left(2\left|e_{1}\right|^{2 q},\left|e_{2}\right|^{2 q}\right)}}\right\} .
\end{gathered}
$$

Proof. Taking $\lambda=m=1, \gamma_{1}=\gamma_{2}=0, \eta\left(e_{2}, m e_{1}\right)=e_{2}-m e_{1}, f(t)=\frac{1}{t}$ and $\varphi(t)=t$, in Theorem 2.9, one can obtain the result immediately.

Proposition 3.11. Let $e_{1}, e_{2} \in \mathbb{R} \backslash\{0\}$, where $e_{1}<e_{2}$. Then for $q \geq 1$, the following inequality hold:

$$
\begin{gathered}
\left|\frac{1}{H\left(e_{1}, e_{2}\right)}-\frac{1}{L\left(e_{1}, e_{2}\right)}\right| \leq \sqrt[q]{\frac{5}{3} \frac{\left(e_{2}-e_{1}\right)}{8}} \\
\times\left\{\frac{1}{\sqrt[q]{H\left(\left|e_{1}\right|^{2 q}, 5\left|e_{2}\right|^{2 q}\right)}}+\frac{1}{\sqrt[q]{H\left(5\left|e_{1}\right|^{2 q},\left|e_{2}\right|^{2 q}\right)}}\right\} .
\end{gathered}
$$

Proof. Taking $\lambda=m=1, \gamma_{1}=\gamma_{2}=1, \eta\left(e_{2}, m e_{1}\right)=e_{2}-m e_{1}, f(t)=\frac{1}{t}$ and $\varphi(t)=t$, in Theorem 2.9, one can obtain the result immediately.

Proposition 3.12. Let $e_{1}, e_{2} \in \mathbb{R} \backslash\{0\}$, where $e_{1}<e_{2}$. Then for $q \geq 1$, the following inequality hold:

$$
\begin{aligned}
\mid \frac{5}{6 H\left(5 e_{1}, e_{2}\right)} & +\frac{1}{2 A\left(e_{1}, e_{2}\right)}-\frac{1}{L\left(e_{1}, e_{2}\right)} \mid \leq\left(\frac{13}{72}\right)^{1-\frac{1}{q}} \frac{\left(e_{2}-e_{1}\right)}{2} \\
& \times\left\{\sqrt[q]{\frac{49715}{1296}} \frac{1}{\sqrt[q]{H\left(163\left|e_{1}\right|^{2 q}, 305\left|e_{2}\right|^{2 q}\right)}}\right. \\
+ & \left.\sqrt[q]{\frac{2300975}{10368}} \frac{1}{\sqrt[q]{H\left(1550\left|e_{1}\right|^{2 q}, 5938\left|e_{2}\right|^{2 q}\right)}}\right\} .
\end{aligned}
$$

Proof. Taking $\lambda=m=1, \gamma_{1}=\frac{1}{6}, \gamma_{2}=\frac{5}{6}, \eta\left(e_{2}, m e_{1}\right)=e_{2}-m e_{1}, f(t)=\frac{1}{t}$ and $\varphi(t)=t$, in Theorem 2.9, one can obtain the result immediately.

Remark 3.13. Applying our Theorems 2.3 and 2.9 for special values of parameter $\lambda, \gamma_{1}$ and $\gamma_{2}$, for appropriate choices of function

$$
\varphi(t)=\frac{t^{\alpha}}{\Gamma(\alpha)}, \frac{t^{\frac{\alpha}{k}}}{k \Gamma_{k}(\alpha)} ; \quad \varphi(t)=t\left(e_{2}-t\right)^{\alpha-1}
$$

for $\alpha \in(0,1) ; \varphi(t)=\frac{t}{\alpha} \exp \left[\left(-\frac{1-\alpha}{\alpha}\right) t\right]$ for $\alpha \in(0,1)$, such that $\left|f^{\prime}\right|^{q}$ to be convex, we can deduce some new general fractional integral inequalities using above special means. The details are left to the interested reader. 
Next, we provide some new error estimates for the midpoint and trapezium quadrature formula. Let $Q$ be the partition of the points $e_{1}=x_{0}<x_{1}<\ldots<x_{k}=e_{2}$ of the interval $\left[e_{1}, e_{2}\right]$. Let consider the following quadrature formula:

$$
\int_{e_{1}}^{e_{2}} f(x) d x=M(f, Q)+E(f, Q), \quad \int_{e_{1}}^{e_{2}} f(x) d x=T(f, Q)+E^{*}(f, Q)
$$

where

$$
M(f, Q)=\sum_{i=0}^{k-1} f\left(\frac{x_{i}+x_{i+1}}{2}\right)\left(x_{i+1}-x_{i}\right)
$$

and

$$
T(f, Q)=\sum_{i=0}^{k-1} \frac{f\left(x_{i}\right)+f\left(x_{i+1}\right)}{2}\left(x_{i+1}-x_{i}\right)
$$

are the midpoint and trapezium version and $E(f, Q), E^{*}(f, Q)$ are denote their associated approximation errors.

Proposition 3.14. Let $f:\left[e_{1}, e_{2}\right] \longrightarrow \mathbb{R}$ be a differentiable function on $\left(e_{1}, e_{2}\right)$, where $e_{1}<e_{2}$. If $\left|f^{\prime}\right|^{q}$ is convex on $\left[e_{1}, e_{2}\right]$ for $q>1$ and $p^{-1}+q^{-1}=1$, then the following inequality holds:

$$
\begin{gathered}
|E(f, Q)| \leq \frac{\sqrt[p]{2}}{2 \sqrt[q]{8} \sqrt[p]{2^{p+1}(p+1)}} \times \sum_{i=0}^{k-1}\left(x_{i+1}-x_{i}\right)^{2} \\
\times\left\{\sqrt[q]{\left|f^{\prime}\left(x_{i}\right)\right|^{q}+3\left|f^{\prime}\left(x_{i+1}\right)\right|^{q}}+\sqrt[q]{3\left|f^{\prime}\left(x_{i}\right)\right|^{q}+\left|f^{\prime}\left(x_{i+1}\right)\right|^{q}}\right\} .
\end{gathered}
$$

Proof. Applying Theorem 2.3 for $\lambda=m=1, \gamma_{1}=\gamma_{2}=0, \eta\left(e_{2}, m e_{1}\right)=e_{2}-m e_{1}$ and $\varphi(t)=t$ on the subintervals $\left[x_{i}, x_{i+1}\right](i=0, \ldots, k-1)$ of the partition $Q$, we have

$$
\begin{gathered}
\left|f\left(\frac{x_{i}+x_{i+1}}{2}\right)-\frac{1}{x_{i+1}-x_{i}} \int_{x_{i}}^{x_{i+1}} f(x) d x\right| \leq \frac{\sqrt[p]{2}\left(x_{i+1}-x_{i}\right)}{2 \sqrt[q]{8} \sqrt[p]{2^{p+1}(p+1)}} \\
\times\left\{\sqrt[q]{\left|f^{\prime}\left(x_{i}\right)\right|^{q}+3\left|f^{\prime}\left(x_{i+1}\right)\right|^{q}}+\sqrt[q]{3\left|f^{\prime}\left(x_{i}\right)\right|^{q}+\left|f^{\prime}\left(x_{i+1}\right)\right|^{q}}\right\} .
\end{gathered}
$$

Hence from (3.14), we get

$$
\begin{gathered}
|E(f, Q)|=\left|\int_{e_{1}}^{e_{2}} f(x) d x-M(f, Q)\right| \\
\leq\left|\sum_{i=0}^{k-1}\left\{\int_{x_{i}}^{x_{i+1}} f(x) d x-f\left(\frac{x_{i}+x_{i+1}}{2}\right)\left(x_{i+1}-x_{i}\right)\right\}\right| \\
\leq \sum_{i=0}^{k-1}\left|\left\{\int_{x_{i}}^{x_{i+1}} f(x) d x-f\left(\frac{x_{i}+x_{i+1}}{2}\right)\left(x_{i+1}-x_{i}\right)\right\}\right| \\
\leq \frac{\sqrt[p]{2}}{2 \sqrt[q]{8} \sqrt[p]{2^{p+1}(p+1)}} \times \sum_{i=0}^{k-1}\left(x_{i+1}-x_{i}\right)^{2} \\
\times\left\{\sqrt[q]{\left|f^{\prime}\left(x_{i}\right)\right| q+3\left|f^{\prime}\left(x_{i+1}\right)\right|^{q}}+\sqrt[q]{3\left|f^{\prime}\left(x_{i}\right)\right|^{q}+\left|f^{\prime}\left(x_{i+1}\right)\right|^{q}}\right\} .
\end{gathered}
$$


The proof of this proposition is complete.

Proposition 3.15. Let $f:\left[e_{1}, e_{2}\right] \longrightarrow \mathbb{R}$ be a differentiable function on $\left(e_{1}, e_{2}\right)$, where $e_{1}<e_{2}$. If $\left|f^{\prime}\right|^{q}$ is convex on $\left[e_{1}, e_{2}\right]$ for $q>1$ and $p^{-1}+q^{-1}=1$, then the following inequality holds:

$$
\begin{gathered}
|E(f, Q)| \leq \frac{1}{2 \sqrt[q]{8} \sqrt[p]{2(p+1)}} \times \sum_{i=0}^{k-1}\left(x_{i+1}-x_{i}\right)^{2} \\
\times\left\{\sqrt[q]{\left|f^{\prime}\left(x_{i}\right)\right|^{q}+3\left|f^{\prime}\left(x_{i+1}\right)\right|^{q}}+\sqrt[q]{3\left|f^{\prime}\left(x_{i}\right)\right|^{q}+\left|f^{\prime}\left(x_{i+1}\right)\right|^{q}}\right\} .
\end{gathered}
$$

Proof. The proof is analogous as to that of Proposition 3.14 taking $\lambda=m=1$, $\gamma_{1}=\gamma_{2}=1, \eta\left(e_{2}, m e_{1}\right)=e_{2}-m e_{1}$ and $\varphi(t)=t$.

Proposition 3.16. Let $f:\left[e_{1}, e_{2}\right] \longrightarrow \mathbb{R}$ be a differentiable function on $\left(e_{1}, e_{2}\right)$, where $e_{1}<e_{2}$. If $\left|f^{\prime}\right|^{q}$ is convex on $\left[e_{1}, e_{2}\right]$ for $q \geq 1$, then the following inequality holds:

$$
\begin{gathered}
\left|E^{*}(f, Q)\right| \leq \frac{1}{8 \sqrt[q]{3}} \times \sum_{i=0}^{k-1}\left(x_{i+1}-x_{i}\right)^{2} \\
\times\left\{\sqrt[q]{\left|f^{\prime}\left(x_{i}\right)\right|^{q}+2\left|f^{\prime}\left(x_{i+1}\right)\right|^{q}}+\sqrt[q]{2\left|f^{\prime}\left(x_{i}\right)\right|^{q}+\left|f^{\prime}\left(x_{i+1}\right)\right|^{q}}\right\} .
\end{gathered}
$$

Proof. Applying Theorem 2.9 for $\lambda=m=1, \gamma_{1}=\gamma_{2}=0, \eta\left(e_{2}, m e_{1}\right)=e_{2}-m e_{1}$ and $\varphi(t)=t$ on the subintervals $\left[x_{i}, x_{i+1}\right](i=0, \ldots, k-1)$ of the partition $Q$, we have

$$
\begin{aligned}
& \left|\frac{f\left(x_{i}\right)+f\left(x_{i+1}\right)}{2}-\frac{1}{x_{i+1}-x_{i}} \int_{x_{i}}^{x_{i+1}} f(x) d x\right| \leq \frac{\left(x_{i+1}-x_{i}\right)}{\sqrt[q]{3}} \\
& \times\left\{\sqrt[q]{\left|f^{\prime}\left(x_{i}\right)\right|^{q}+2\left|f^{\prime}\left(x_{i+1}\right)\right|^{q}}+\sqrt[q]{2\left|f^{\prime}\left(x_{i}\right)\right|^{q}+\left|f^{\prime}\left(x_{i+1}\right)\right|^{q}}\right\} .
\end{aligned}
$$

Hence from (3.17), we get

$$
\begin{gathered}
\left|E^{*}(f, Q)\right|=\left|\int_{e_{1}}^{e_{2}} f(x) d x-T(f, Q)\right| \\
\leq\left|\sum_{i=0}^{k-1}\left\{\int_{x_{i}}^{x_{i+1}} f(x) d x-\frac{f\left(x_{i}\right)+f\left(x_{i+1}\right)}{2}\left(x_{i+1}-x_{i}\right)\right\}\right| \\
\leq \sum_{i=0}^{k-1}\left|\left\{\int_{x_{i}}^{x_{i+1}} f(x) d x-\frac{f\left(x_{i}\right)+f\left(x_{i+1}\right)}{2}\left(x_{i+1}-x_{i}\right)\right\}\right| \\
\leq \frac{1}{8 \sqrt[q]{3}} \times \sum_{i=0}^{k-1}\left(x_{i+1}-x_{i}\right)^{2} \\
\times\left\{\sqrt[q]{\left|f^{\prime}\left(x_{i}\right)\right|^{q}+2\left|f^{\prime}\left(x_{i+1}\right)\right|^{q}}+\sqrt[q]{2\left|f^{\prime}\left(x_{i}\right)\right|^{q}+\left|f^{\prime}\left(x_{i+1}\right)\right|^{q}}\right\} .
\end{gathered}
$$

The proof of this proposition is complete. 
Proposition 3.17. Let $f:\left[e_{1}, e_{2}\right] \longrightarrow \mathbb{R}$ be a differentiable function on $\left(e_{1}, e_{2}\right)$, where $e_{1}<e_{2}$. If $\left|f^{\prime}\right|^{q}$ is convex on $\left[e_{1}, e_{2}\right]$ for $q \geq 1$, then the following inequality holds:

$$
\begin{gathered}
\left|E^{*}(f, Q)\right| \leq \frac{1}{8 \sqrt[q]{6}} \times \sum_{i=0}^{k-1}\left(x_{i+1}-x_{i}\right)^{2} \\
\times\left\{\sqrt[q]{\left|f^{\prime}\left(x_{i}\right)\right|^{q}+5\left|f^{\prime}\left(x_{i+1}\right)\right|^{q}}+\sqrt[q]{5\left|f^{\prime}\left(x_{i}\right)\right|^{q}+\left|f^{\prime}\left(x_{i+1}\right)\right|^{q}}\right\} .
\end{gathered}
$$

Proof. The proof is analogous as to that of Proposition 3.16 taking $\lambda=m=1$, $\gamma_{1}=\gamma_{2}=1, \eta\left(e_{2}, m e_{1}\right)=e_{2}-m e_{1}$ and $\varphi(t)=t$.

Remark 3.18. Applying our Theorems 2.3 and 2.9, where $m=1$, for special values of parameter $\lambda, \gamma_{1}$ and $\gamma_{2}$, for appropriate choices of function

$$
\begin{gathered}
\varphi(t)=\frac{t^{\alpha}}{\Gamma(\alpha)}, \frac{t^{\frac{\alpha}{k}}}{k \Gamma_{k}(\alpha)} \\
\varphi(t)=t\left(e_{2}-t\right)^{\alpha-1}
\end{gathered}
$$

for $\alpha \in(0,1)$;

$$
\varphi(t)=\frac{t}{\alpha} \exp \left[\left(-\frac{1-\alpha}{\alpha}\right) t\right]
$$

for $\alpha \in(0,1)$, such that $\left|f^{\prime}\right|^{q}$ to be convex, we can deduce some new bounds for the midpoint and trapezium quadrature formula using above ideas and techniques. The details are left to the interested reader.

Acknowledgements. The authors would like to thank the referee for valuable comments and suggestions.

\section{References}

[1] Aslani, S.M., Delavar, M.R., Vaezpour, S.M., Inequalities of Fejér type related to generalized convex functions with applications, Int. J. Anal. Appl., 16(2018), no. 1, 38-49.

[2] Chen, F.X., Wu, S.H., Several complementary inequalities to inequalities of HermiteHadamard type for s-convex functions, J. Nonlinear Sci. Appl., 9(2016), no. 2, 705-716.

[3] Chu, Y.M., Khan, M.A., Khan, T.U., Ali, T., Generalizations of Hermite-Hadamard type inequalities for MT-convex functions, J. Nonlinear Sci. Appl., 9(2016), no. 5, 4305-4316.

[4] Delavar, M.R., Dragomir, S.S., On $\eta$-convexity, Math. Inequal. Appl., 20(2017), 203-216.

[5] Delavar, M.R., De La Sen, M. Some generalizations of Hermite-Hadamard type inequalities, Springer Plus, 5(2016), no. 1661.

[6] Dragomir, S.S., Agarwal, R.P., Two inequalities for differentiable mappings and applications to special means of real numbers and trapezoidal formula, Appl. Math. Lett., 11(1998), no. 5, 91-95.

[7] Farid, G., Rehman, A.U., Generalizations of some integral inequalities for fractional integrals, Ann. Math. Sil., 31(2017), pp. 14.

[8] Kashuri, A., Liko, R., Some new Hermite-Hadamard type inequalities and their applications, Stud. Sci. Math. Hung., 56(2019), no. 1, 103-142. 
[9] Khan, M.A., Chu, Y.M., Kashuri, A., Liko, R., Hermite-Hadamard type fractional integral inequalities for $M T_{(r ; g, m, \phi)}$-preinvex functions, J. Comput. Anal. Appl., 26(2019), no. 8, 1487-1503.

[10] Khan, M.A., Chu, Y.M., Kashuri, A., Liko, R., Ali, G., New Hermite-Hadamard inequalities for conformable fractional integrals, J. Funct. Spaces, (2018), Article ID 6928130, pp. 9.

[11] Liu, W.J., Some Simpson type inequalities for $h$-convex and $(\alpha, m)$-convex functions, J. Comput. Anal. Appl., 16(2014), no. 5, 1005-1012.

[12] Liu, W., Wen, W., Park, J., Hermite-Hadamard type inequalities for MT-convex functions via classical integrals and fractional integrals, J. Nonlinear Sci. Appl., 9(2016), 766-777.

[13] Luo, C., Du, T.S., Khan, M.A., Kashuri, A., Shen, Y., Some k-fractional integrals inequalities through generalized $\lambda_{\phi m}-M T$-preinvexity, J. Comput. Anal. Appl., 27(2019), no. 4, 690-705.

[14] Mihai, M.V., Some Hermite-Hadamard type inequalities via Riemann-Liouville fractional calculus, Tamkang J. Math, 44(2013), no. 4, 411-416.

[15] Mubeen, S., Habibullah, G.M., k-Fractional integrals and applications, Int. J. Contemp. Math. Sci., 7(2012), 89-94.

[16] Omotoyinbo, O., Mogbodemu, A., Some new Hermite-Hadamard integral inequalities for convex functions, Int. J. Sci. Innovation Tech., 1(2014), no. 1, 1-12.

[17] Özdemir, M.E., Dragomir, S.S., Yildiz, C., The Hadamard's inequality for convex function via fractional integrals, Acta Math. Sci., Ser. A, Chin. Ed., 33(2013), no. 5, 153-164.

[18] Qi, F., Xi, B.Y., Some integral inequalities of Simpson type for $G A-\epsilon$-convex functions, Georgian Math. J., 20(2013), no. 5, 775-788.

[19] Sarikaya, M.Z., Ertuğral, F., On the generalized Hermite-Hadamard inequalities, https://www.researchgate.net/publication/321760443.

[20] Sarikaya, M.Z., Yildirim, H., On generalization of the Riesz potential, Indian Jour. of Math. and Mathematical Sci., 3(2007), no. 2, 231-235.

[21] Set, E., Noor, M.A., Awan, M.U., Gözpinar, A., Generalized Hermite-Hadamard type inequalities involving fractional integral operators, J. Inequal. Appl., 169(2017), 1-10.

[22] Wang, H., Du, T.S., Zhang, Y., k-fractional integral trapezium-like inequalities through $(h, m)$-convex and $(\alpha, m)$-convex mappings, J. Inequal. Appl., 2017(2017), no. 311, pp. 20 .

[23] Xi, B.Y., Qi, F., Some integral inequalities of Hermite-Hadamard type for convex functions with applications to means, J. Funct. Spaces Appl., 2012(2012), Article ID 980438, pp. 14.

[24] Zhang, X.M., Chu, Y.M., Zhang, X.H., The Hermite-Hadamard type inequality of GAconvex functions and its applications, J. Inequal. Appl., (2010), Article ID 507560, pp. 11.

[25] Zhang, Y., Du, T.S., Wang, H., Shen, Y.J., Kashuri, A., Extensions of different type parameterized inequalities for generalized $(m, h)$-preinvex mappings via $k$-fractional integrals, J. Inequal. Appl., 2018(2018), no. 49, pp. 30. 
Artion Kashuri

Department of Mathematics,

Faculty of Technical Science,

University Ismail Qemali,

Vlora, Albania

e-mail: artionkashuri@gmail.com

Rozana Liko

Department of Mathematics, Faculty of Technical Science, University Ismail Qemali, Vlora, Albania

e-mail: rozanaliko86@gmail.com 